\title{
Central and peripheral visual processing in hearing and nonhearing individuals
}

\author{
WING HONG LOKE and SHAREEN SONG \\ National University of Singapore, Singapore
}

\begin{abstract}
Hearing and nonhearing students responded to stimuli presented in their central and peripheral visual fields. No significant difference was found between the reaction times of hearing and nonhearing students in the central visual field condition. However, in the peripheral visual field condition, nonhearing students were significantly faster than hearing students. Hearing-impaired students may have developed a more alert peripheral vision. Perhaps, as a result of their auditory impairment, nonhearing students make greater use of visual communication and need to monitor new information more via the visual channel. Specific suggestions for future research on the development of peripheral vision are discussed.
\end{abstract}

According to Wundt (1911/1973), "the keenest differentiation of impressions is limited to the so-called region of clearest vision, which surrounds the fixation point"' (p. 18). This region, known as the fovea, has the greatest concentration of rod and cone cells, so visual acuity and alertness are highest for the viewing of stimuli presented in the central visual field. As the density of visual cells decreases with distance from the fovea, the visual acuity and alertness also decrease for stimuli presented in the peripheral field. Past studies (Crassini, Brown, \& Bowman, 1988; Posner \& Davidson, 1976) have demonstrated visual superiority of the fovea (i.e., faster reaction time and greater contrast sensitivity) over the parafovea.

Of particular interest is that congenitally deaf people may differ from hearing people in development of the attentional system, owing to novel constraints imposed by the requirement to develop a visuospatial language system (Parasnis \& Samar, 1985). The use of sign language (e.g., the American Sign Language, or ASL, which is also used in Singapore) generally requires a sign space that extends beyond the receiver's foveal and parafoveal fields. A receiver fluent in sign language visually fixates on the face or lips of the signer during communication. Linguistic information is conveyed by the use of hands and lips and by facial expression, so the information is distributed over a broad spatial area. In addition, certain linguistic features of ASL depend on the direct manipulation of the receiver's attention to specific locations in the sign space.

This research was supported by National University of Singapore Grant RP89003 awarded to W. H. Loke. The authors thank Goh Tong Pak, Ong Poh Huat, Rebecca Pakiam, Chua Siew Suan, Lim Chin Heng, Daniel Gwee, and Judy Ho for their assistance and J. E. Bellquist for his editorial comments. Correspondence should be addressed to W. $\mathbf{H}$. Loke, Department of Social Work and Psychology, 10 Kent Ridge Crescent, National University of Singapore, Singapore 0511.
To illustrate the complex spatiotemporal structure of the language, Parasnis and Samar (1985) note:

\begin{abstract}
When making pronominal reference to two different persons not present (as in a "then he said, then she said" report), the signer often will initially tag a spatial reference point off to the right for one person, and off to the left for another, using the person classifier after naming that person. Pronominal reference is then made by pointing to these locations, and reported dialogue is often accomplished by rapidly shifting the locus of manual articulation and body posture toward those spatial locations.... This complex spatiotemporal language structure, coupled with the tendency of the receiver to fixate on the face and lips, suggests that signed communication demands a more dynamic modulation of attention to different parts of the visual field during the normal course of reception than does oral communication. (para. 2, p. 314)
\end{abstract}

In addition, deaf people rely on their vision for environmental cues, in contrast with hearing people, who receive environmental feedback from the auditory channel. In particular, the hearing-impaired may possess greater peripheral acuity because they constantly monitor the peripheral visual field for new information. Hearing people, however, rely on both the visual periphery and the auditory sense to monitor new information (Parasnis \& Samar, 1985). Consider these two situations: (1) When one wants to attract the attention of a deaf person a few meters away, the appropriate method is to wave in the direction of the person; conversely, if the attention of a hearing person is needed, one simply yells. (2) A deaf driver would have to rely on peripheral vision to perceive that a car is approaching from behind at great speed, whereas a hearing individual relies on the sound of the approaching engine or the blast of the horn.

In most studies only hearing individuals have been tested, although in a few studies (e.g., Parasnis \& Samar, 1985) hearing and nonhearing individuals have been compared. Parasnis and Samar showed that both deaf and hearing 
young adults utilize cues to direct attention to specific locations and have difficulty in ignoring foveal information. However, deaf people were more efficient in redirecting information from one spatial location to another in the presence of irrelevant foveal information, suggesting differences in the development of attention mechanisms in hearing and nonhearing individuals. In the present study, we compared the visual alertness of hearing and nonhearing individuals in response to the presentation of stimuli in the peripheral as well as the central visual fields. Hearing and nonhearing 14- to 20-year-old secondary school students were tested. The nonhearing were congenitally deaf or had been profoundly deaf for 12 or more years, and they were ASL signers. A computer was used to present the stimuli in the subject's central or peripheral visual field and to measure their reaction times. Although several studies have been done in this way, in most studies the stimuli have been presented with a tachistoscope. We predicted that the nonhearing individuals would possess greater alertness in response to stimuli presented in the peripheral visual field than hearing people would, but that when stimuli were presented in the central visual field, visual alertness would be similar (nonsignificant) in the hearing and nonhearing subjects. All individuals utilize their foveal vision to monitor for new information.

\section{METHOD}

\section{Subjects}

Nineteen hearing students ( 11 males and 8 females; mean age, 15.2 years; range, 14-17 years) and 20 nonhearing students ( 9 males and 11 females; mean age, 16.7 years; range, 15-20 years) participated. They attended a school with a curriculum for the hearing-impaired. Thirteen of the nonhearing subjects were congenitally deaf, 6 had lost their hearing through an illness before they were 1 year old, and 1 had lost hearing in a fall at the age of 5 . All of the nonhearing subjects were either profoundly or totally deaf, and most were not in the habit of wearing hearing aids.

\section{Apparatus and Stimuli}

A computer program presented the stimuli and recorded the data. For each test trial, a random numeral ranging from 1 to 9 (the fixation number) appeared at the middle of the screen. After a randomly determined time lapse of 2-7 sec, an asterisk (the stimulus) was shown on the screen. The asterisk was presented in either the central or the peripheral visual field of the subject. In the central visual field condition, this stimulus was presented near the random number (less than $0.5^{\circ}$ from fixation). In the peripheral visual field condition, the stimulus was presented farther away ( $25^{\circ}$ from fixation). The subjects were instructed to respond by pressing the space bar as soon as they perceived the stimulus. The screen was then cleared, and the subjects were prompted by the computer to type in the number that had appeared on the screen. The trial ended after the subject had identified the number; the appearance of another number at the middle of the screen marked the beginning of the next trial. If a subject responded before the asterisk appeared, a reminder to the subject to respond only after the onset of the stimulus was presented at the bottom of the screen. Then the next trial began (see Figure 1). It should be noted that Posner and colleagues (Posner \& Davidson, 1976; Posner, Nissen, \& Ogden, 1978) found the costs and benefits for foveal and peripheral stimuli to be similar, implying that when attention is moved to a peripheral location, the area of most efficient detection is moved so that it is symmetric around the expected location (Posner, 1986). So to minimize the possibility that subjects would shift their locus of attention (i.e., the central visual field), they were given a number to fixate, and they were subsequently asked to recall it. Because the subjects were required to respond to the stimulus as soon

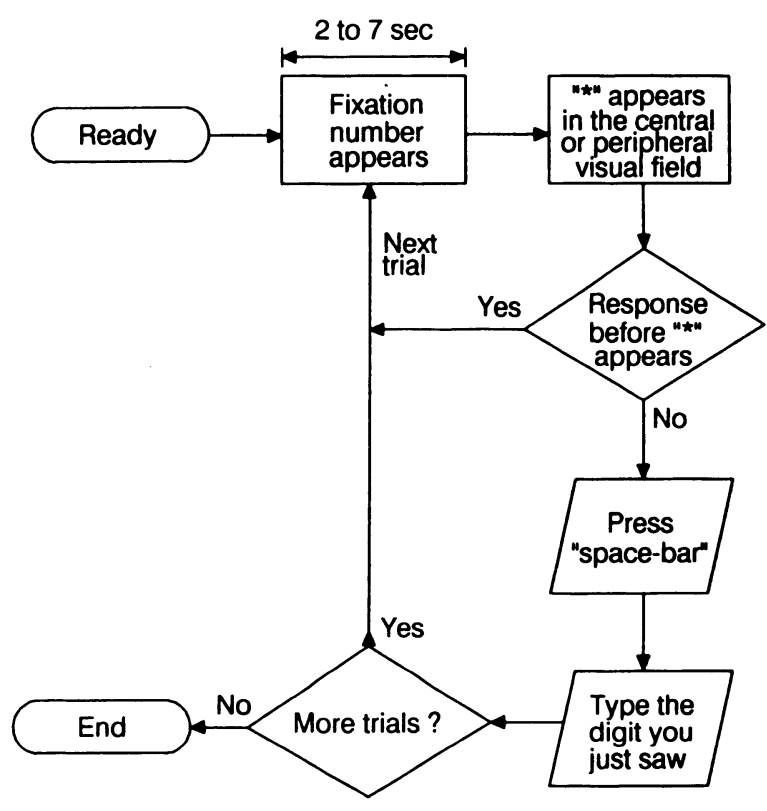

Figure 1. Frame sequence of a trial presented on the computer. The duration of the two response frames $(\square)$ depends on the subject's response time.

as they had perceived its appearance, they would not shift their fixation to the stimulus.

Each reaction time for the correct identification of the fixation number was recorded and used for subsequent analyses of central as opposed to peripheral visual field conditions. The calculation of the average excluded trials on which the subject had incorrectly identified the fixation number or had responded before the onset of the stimulus. Twenty trials, 10 for the central condition and 10 for the peripheral visual field condition, were presented in a random manner. Each trial took an average of $15 \mathrm{sec}$, and the entire experiment (test trials, briefing, and debriefing procedures) lasted for about $8 \mathrm{~min}$.

\section{Pilot Testing}

Pilot testing was conducted on 10 undergraduates from the National University of Singapore to determine whether the computer program was effective in showing a difference between scores in the central and peripheral visual field conditions. We also examined the positioning of the headrest and tested the use of an eyepatch for testing monocular vision. The findings showed that when the central and peripheral stimuli were presented, respectively, at $0.5^{\circ}$ and $25^{\circ}$ from the fixation point, the eye should be aligned $20 \mathrm{~cm}$ away from the center of the monitor screen. The eyepatch caused some stiff necks and discomfort to the eyes. Also, the number of trials was varied across subjects so that an attentional threshold for the task could be determined. Eventually, a suitable positioning of the headrest and eyepatch and a suitable number of trials were determined. We selected 20 trials; with more than that, subjects experienced tedium and their attentions waned. The central and peripheral visual field conditions each consisted of 10 trials.

As expected, the pilot results showed that subjects had faster reaction times in the central than in the peripheral condition; the mean times were 385 and $453 \mathrm{msec}$, respectively. No inferential analysis was done because of the small sample size.

\section{Procedure}

A laptop (10-MHz with coprocessor) computer was brought to a local secondary school on 3 consecutive school days and was set up on a table in the corner of a classroom. A headrest was placed in front of the computer to minimize the head movement of the subjects and to ensure that the subjects positioned their heads about $20 \mathrm{~cm}$ away from the computer screen. 
The subjects were tested individually. Each subject was briefed on the experimental task and given a demonstration. The subject then received a practice run of six trials. The experimenter provided verbal instructions in ASL for the nonhearing subjects.

The subject was then taught to identify the dominant eye in the following manner: Each subject pointed at an object (about $3 \mathrm{~m}$ away) and looked at it through one eye at a time. The dominant eye was identified as that which still viewed the object in line with the tip of the finger. All subjects were able to identify their dominant eyes with this procedure, except for 1 nonhearing female. Since more ( 26 out of 39 ) subjects had a right dominant eye, she was told to do the experimental task with her right eye. She was not excluded from the study, for her eyesight was normal.

After the subject had determined the dominant eye, an eyepatch was used to cover the other eye. Then, three rules were repeated to the subject: (1) The subject was to focus the dominant eye at the fixation number presented at the start of each trial; (2) the space bar was to be pressed as soon as the subject saw the asterisk on the screen; and (3) before each trial, the subject was to rest (not press) the thumb and fingers of the dominant hand on the space bar.

After the experiment, each subject was given a piece of candy and debriefed. A contact number and address were given to the teachers in the school in case of further questions.

\section{RESULTS}

In general, subjects responded faster when the stimuli were presented in the central rather than the peripheral visual field [means of 454 vs. $513 \mathrm{msec}$, respectively; $F(1,39)=20.08, p<.001]$. For the central visual field condition, the difference in reaction times between hearing and nonhearing subjects was nonsignificant [means of 474 and $436 \mathrm{msec}$, respectively; $F(1,38)=1.86$, $p=.18$ ]. In contrast, in the peripheral visual field condition, the nonhearing subjects responded faster than the hearing subjects [means of 472 and $557 \mathrm{msec}$, respectively; $F(1,38)=4.85, p<.03$ ].

In addition, the difference in the reaction times between the central and peripheral conditions was calculated for the hearing and nonhearing subjects. This difference reflects the attentional differences minus the speed to react to the presentation of the stimulus. In other words, subjects in the central condition "react" to the appearance of the stimuli, whereas in the peripheral condition they have to "detect" and then "react" to the stimuli. So the calculated difference reflects time needed to "detect" the appearance of the stimuli, partialling out the time to "react." The hearing subjects showed a greater difference in reaction time than the nonhearing subjects [means of 83 vs. $36 \mathrm{msec}$, respectively; $F(1,38)=3.54, p<$ .06]. This suggests that the hearing group took more time to "detect" the appearance of the stimuli; or in simpler terms, they were less attentive than the nonhearing subjects. Altogether, the results suggest that the nonhearing subjects have a superior attentional system for peripheral information as opposed to central information.

Several secondary analyses were also conducted. A correlational test was used to examine whether the subjects' age correlated with their reaction times. In the present study, the mean age of the nonhearing subjects was 1.5 years greater than that of the hearing subjects. Results showed nonsignificant correlations for both the central $(r=-.07)$ and peripheral $(r=-.04)$ visual field con- ditions. In addition, differences between males and females were examined. There were no significant gender differences in reaction times in the central condition $[t(17)$ $=0.98$ for the hearing subjects, and $t(18)=0.31$ for the nonhearing subjects] and in the peripheral condition $[t(17)$ $=0.89$ for the hearing subjects, and $t(17)=0.17$ for the nonhearing subjects].

\section{DISCUSSION}

The present study is consistent with previous studies of hearing individuals (e.g. . Posner \& Davidson, 1976) in that hearing and nonhearing subjects responded significantly faster when the stimuli were presented in the central rather than the peripheral visual field. In addition, nonhearing subjects responded faster than nonhearing subjects in the peripheral but not the central condition. Although hearing and nonhearing subjects do not differ in foveal (central) reflexes, nonhearing individuals have superior peripheral vision. Hearing and nonhearing individuals "react" equally well to stimuli in the central visual field, because the act of "reacting" requires just seeing the stimuli that are in the individual's direct line of sight.

For the peripheral visual field condition, there are two plausible explanations for the higher performance of the nonhearing as opposed to the hearing subjects. First, the development of better peripheral vision may be due to the hearing handicap of nonhearing subjects, which necessitates reliance on the visual sense to process new information. Just as an unexpected sound or the calling of one's own name, the sudden appearance of an individual or an object in the visual periphery can serve as an alerting stimulus. Because nonhearing individuals lack the normal functions of audition, they depend on other senses such as peripheral vision to evoke orienting responses to these events. The dependence on the visual periphery eventually leads to more efficient monitoring and orienting mechanisms in visual attention (Parasnis \& Samar, 1985).

The second explanation involves the use of sign language as a means of communication. Because the signing space often stretches beyond the area that can be monitored by foveal vision, the receiver has to use the visual periphery to "decode" the signs. This constant utilization of the visual periphery during communication may facilitate the receiver's alertness to it. In future research, the significance of a visual language should be investigated through comparison of hearing individuals who know and use sign language with those who do not. Can the development of parafoveal vision be enhanced by regular use of the visual periphery (in the use of sign language) or through constant dependence (such as the need to constantly monitor parafoveal information by the hearing individuals)? Posttest interviews revealed that most of the hearing subjects knew some sign language (the alphabet and some basic signs) and communicated with their nonhearing friends by means of a mixture of ASL, gestures, and facial expressions. Despite the ability to communicate with (minimal) visual language, they performed significantly slower than their nonhearing counterparts in the peripheral visual field condition. In future studies, subjects' level of fluency in and use of sign language should therefore be controlled.

The development of superior peripheral vision in nonhearing individuals may also owe to the fact that hearing people do not (frequently) use the peripheral visual field. In many cultures, children are taught to focus their attention on certain tasks. For example, reading and writing require individuals to focus attention in the designated (spatial) area. To attend to events in the visual periphery is discouraged because it affects the task performance. Thus, hearing individuals learn to develop foveal information first, which leads to the relative deterioration of their peripheral vision. Holmes, Cohen, Haith, and Morrison (1977) studied the peripheral vision of children and adults by having them report items presented in the visual and peripheral fields simultaneously. The results indicated that 5-year-old children do not show any pronounced effects of condition whereas 8-year-olds and adults tend to report foveal items first (this tendency increases with age). Holmes et al. (1977) suggested that this automatic fovea-first processing strategy might be learned, because it was not present in the younger children. In the present study, hearing individuals had greater differences in reaction times between the peripheral and central visual field conditions than nonhearing in- 
dividuals did. The implication is that there are smaller attentional differences between the central and peripheral vision in nonhearing individuals (similar to the 5-year-olds in Holmes et al.'s, 1977, study), whereas such differences are greater in the hearing subjects. Hence, the learned fovea-first processing strategy in hearing individuals may also have contributed to the different effects of the visual periphery in hearing and nonhearing individuals (in addition to deaf people's developing a superior peripheral vision).

The potential scope of research involving hearing-impaired subjects is wide. More could be learned about the development of peripheral vision, and in particular about the development of the peripheral visual system that results from constant use and dependence. Are there any physiological differences in the retina of hearing as opposed to nonhearing individuals? More extensive research should be done to compare other visual processes in hearing and deaf individuals. From an applied perspective, studies on hearing-impaired individuals can perhaps yield results that could suggest occupational specializations suitable for the hearing-impaired. For example, given that the hearing-impaired have better ability to perceive visual information than hearing individuals do, they could be trained for jobs that require detection of stimuli in the visual periphery. Rather than consider only the negative aspects of such a handicap, employers of nonhearing individuals might consider the "advantages" of such impairment. Having more alert peripheral vision, deaf individuals would be faster to detect whether something has gone awry in an environment in which no sound signal is given. The significant but mere 85 -msec faster response time of nonhearing individuals (given the limiting conditions of the present study) could translate to a practical significance in the work environment. Furthermore, because deaf individuals have no auditory sense, their work performance should not be impaired by environmental noise. By the same token, they may be suited to supervisory roles in noisy factory settings where sound signals from a faulty machine may be difficult to detect but where an acute visual periphery would enhance quick identification of any such problems.

\section{REFERENCES}

Crassini, B., Brown, B., Bowman, K. (1988). Age-related changes in contrast sensitivity in central and peripheral retina. Perception, 17, 315-332.

Holmes, D. L., Cohen, K. M., Haith, M. M., \&orrison, F. J. (1977). Peripheral visual processing. Perception \& Psychophysics, 22, 571-577.

Parasnis, I., \& Samar, V. J. (1985). Parafoveal attention in congenitally deaf and hearing young adults. Brain \& Cognition, 4, 313-327.

Posner, M. I. (1986). Chronometric explorations of mind. Oxford: Oxford University Press.

Posner, M. I., \& DAvidson, B. J. (1976, July). Automatic and attended components of orienting. Proceedings of the International Congress of Official Physical Activity Sciences, Quebec City. (Mongraph No. 5)

Posner, M. I., Nissen, M. J., OGden, W. C. (1978). Attended and unattended processing models: The role of set for spatial location. In H. L. Pick \& I. J. Saltzman (Eds.), Modes of perceiving and processing information. Hillsdale, NJ: Erlbaum.

WundT, W. (1973). Classics in psychology. New York: Arno Press. (Original work published 1911)

(Manuscript received April 16, 1991.) 